\title{
Developmental pathways from childhood ADHD to adolescent depression: insights from the ALSPAC study
}

\author{
Graeme Fairchild ${ }^{1}$
}

Published online: 9 October 2020

○) Springer-Verlag GmbH Germany, part of Springer Nature 2020

In this editorial, I have chosen to highlight an interesting and important paper published by Powell and colleagues in the current edition of ECAP [1]. There is now compelling evidence that Attention-Deficit/Hyperactivity Disorder (ADHD) in childhood is linked to a range of negative outcomes in adolescence and adulthood [2, 3]. In particular, there seems to be a strong link between childhood ADHD and later depression and suicidal behaviour [4]. However, we know relatively little about the mechanisms that explain this association, although it has been hypothesised that a sense of failure in one's peer relationships or academic settings can lay the foundations for later depression as this leads the young person to develop maladaptive cognitions about the self and low self-esteem [5].

Powell et al. harnessed data from a prospective longitudinal birth cohort study in the UK, the Avon Longitudinal Study of Parents and Children (ALSPAC), in an attempt to address this issue. The authors tested whether the link between early ADHD and later depression was mediated by the detrimental impact of ADHD on academic performance or peer relationships. The authors replicated previous findings obtained in clinical samples that childhood ADHD symptoms (at age 7.5) were related to both higher rates of depressive symptoms and a greater risk for clinically significant depressive symptoms at age 17.5 , but crucially were able to show that both academic attainment at age 16 (assessed using formal examination results) and peer problems at age 16 significantly mediated this association. Peer problems and academic attainment accounted for $15 \%$ and $20 \%$ of the association between ADHD and depressive symptoms respectively. Consequently, this study identifies peer relationships and academic performance as potential prevention or intervention targets amongst children with

Graeme Fairchild

g.fairchild@bath.ac.uk

1 Department of Psychology, University of Bath, Bath BA2 7AY, UK
ADHD. The authors were able to show that their findings were not explained by potentially confounding variables such as parental socioeconomic status or child age and sex. In addition, the association between ADHD and adolescent depression still held when controlling for emotional problems at age 8, suggesting that ADHD came first and led to depressive symptoms at a later stage.

Important strengths of this study include the longitudinal design and the authors' ability to test for mediating effects of peer problems and low academic attainment, thereby shedding light on mechanisms which explain why early ADHD is linked to later depression. The use of a large representative sample increases the likelihood that the findings will be generalisable, in contrast with studies which have relied on clinic-referred samples who are likely to have very high rates of comorbid disorders. This is potentially problematic because common comorbidities of ADHD such as Conduct Disorder, Oppositional Defiant Disorder (ODD) or ODDrelated symptoms such as irritability may be independently related to an increased risk for depression [6-8]. It also highlights the power of the ALSPAC study-few cohort studies have assessed such a comprehensive range of factors and variables such as attainment in national examinations, even though it should be noted that the present study relied on a much smaller sample $(n=2950$ for the analyses testing for longitudinal associations between ADHD and depression and $n=2161$ for the mediation analyses) than was originally recruited at the start of the project $(n=13,988)$. In addition to this high degree of participant attrition, a further limitation was that only a small number of children in the ALSPAC sample fulfilled DSM-IV criteria for ADHD at age 7.5 ( $n=14$; equating to just $0.5 \%$ of the cohort) and rates of clinically diagnosed ADHD and ADHD symptoms were low relative to those reported in other population-based cohorts (e.g., a prevalence rate of $2 \%$ was reported in a large UK study; [9]). Lastly, although depressive symptoms were assessed around 1.5 years after peer relationships and academic attainment were measured, these assessment points 
were actually quite close together, and it is possible that depression may have emerged first and the young person developed peer problems as a consequence (or the relationship between peer problems and depression may be bidirectional, rather than unidirectional). A similar pattern of reverse causation could apply to the links between academic attainment and depression, i.e., emerging depressive symptoms could negatively affect the young person's motivation to engage with education or even impair their memory or executive functions. It has also been shown that depression is associated with poorer school attendance in adolescence [10]. Countering this argument, the prospective relationships between childhood ADHD and late adolescent depression still held when controlling for emotional problems at age 8 or depressive symptoms at age 14 , suggesting that depression did not precede the peer problems or academic difficulties observed in this study.

Overall, these findings suggest that the negative effects of childhood ADHD on academic performance and peer relationships may lead young people with ADHD to feel like failures at school and to find it difficult to form and maintain friendships, thereby leaving them at increased risk for depression. The fact that these mediating factors are potentially modifiable is a hopeful message to emerge from the study. One practical implication of the results is that children with ADHD should be closely monitored for depressive symptoms in adolescence, especially at key transition stages such as leaving formal education. In addition, the findings suggest that social skills training and providing additional support in school might not only benefit children with ADHD in the short term, but could protect them against negative outcomes in the longer term. Future research could examine whether effective treatment for ADHD-either psychological, pharmacological or psychosocial-weakens the association between childhood ADHD and adolescent depression. It would also be interesting to investigate whether certain clusters of ADHD symptoms (e.g., hyperactive or impulsive) are more strongly linked to later depression than others and whether there are differences between persistent versus desisting forms of ADHD in the strength of the association with later depression.

\section{Compliance with ethical standards}

Conflict of interest The author declares that they have no conflict of interest.

\section{References}

1. Powell V, Riglin L, Hammerton G et al (2020) What explains the link between childhood ADHD and adolescent depression? Investigating the role of peer relationships and academic attainment. Eur Child Adolesc Psychiatry. https://doi.org/10.1007/ s00787-019-01463-w

2. Faraone SV, Asherson P, Banaschewski T et al (2015) Attentiondeficit/hyperactivity disorder. Nat Rev Dis Primers 1:15020. https ://doi.org/10.1038/nrdp.2015.20

3. Erskine HE, Norman RE, Ferrari AJ et al (2016) Long-term outcomes of attention-deficit/hyperactivity disorder and conduct disorder: a systematic review and meta-analysis. J Am Acad Child Adolesc Psychiatry 55:841-850. https://doi.org/10.1016/j. jaac.2016.06.016

4. Chronis-Tuscano A, Molina BSG, Pelham WE et al (2010) Very early predictors of adolescent depression and suicide attempts in children with attention-deficit/hyperactivity disorder. Arch Gen Psychiatry 67:1044-1051. https://doi.org/10.1001/archgenpsy chiatry.2010.127

5. Cole DA, Martin JM, Powers B (1997) A competency-based model of child depression: a longitudinal study of peer, parent, teacher, and self-evaluations. J Child Psychol Psychiatry 38:505514. https://doi.org/10.1111/j.1469-7610.1997.tb01537.x

6. Whelan Y, Stringaris A, Maughan B, Barker ED (2013) Developmental continuity of oppositional defiant disorder subdimensions at ages 8,10 , and 13 years and their distinct psychiatric outcomes at age 16 years. J Am Acad Child Adolesc Psychiatry 52:961-969. https://doi.org/10.1016/j.jaac.2013.06.013

7. Fairchild G, Hawes DJ, Frick PJ et al (2019) Conduct Disorder. Nat Rev Dis Primers 5:43. https://doi.org/10.1038/s4157 2-019-0095-y

8. Eyre O, Riglin L, Leibenluft E et al (2019) Irritability in ADHD: association with later depression symptoms. Eur Child Adolesc Psychiatry 28:1375-1384. https://doi.org/10.1007/s00787-01901303-x

9. Ford T, Goodman R, Meltzer H (2003) The British child and adolescent mental health survey 1999: the prevalence of DSM-IV disorders. J Am Acad Child Adolesc Psychiatry 42:1203-1211. https://doi.org/10.1097/00004583-200310000-00011

10. Finning K, Ford T, Moore DA et al (2020) Emotional disorder and absence from school: findings from the 2004 British Child and Adolescent Mental Health Survey. Eur Child Adolesc Psychiatry 29:187-198. https://doi.org/10.1007/s00787-019-01342-4 